\title{
Fractional Integral and Generalized Stieltjes Transforms for Hypergeometric Functions as Transmutation Operators ${ }^{\star}$
}

Tom H. KOORNWINDER

Korteweg-de Vries Institute, University of Amsterdam, P.O. Box 94248, 1090 GE Amsterdam, The Netherlands

E-mail: T.H.Koornwinder@uva.nl

URL: https://staff.fnwi.uva.nl/t.h.koornwinder/

Received April 29, 2015, in final form September 14, 2015; Published online September 20, 2015 http://dx.doi.org/10.3842/SIGMA.2015.074

\begin{abstract}
For each of the eight $n$-th derivative parameter changing formulas for Gauss hypergeometric functions a corresponding fractional integration formula is given. For both types of formulas the differential or integral operator is intertwining between two actions of the hypergeometric differential operator (for two sets of parameters): a so-called transmutation property. This leads to eight fractional integration formulas and four generalized Stieltjes transform formulas for each of the six different explicit solutions of the hypergeometric differential equation, by letting the transforms act on the solutions. By specialization two Euler type integral representations for each of the six solutions are obtained.
\end{abstract}

Key words: Gauss hypergeometric function; Euler integral representation; fractional integral transform; Stieltjes transform; transmutation formula

2010 Mathematics Subject Classification: 33C05; 44A15; 44A20; 26A33

\section{Introduction}

This paper has two sources of inspiration. The first aim was to give a complete list of the fractional integration formulas corresponding to the eight parameter changing $n$-th derivative formulas for Gauss hypergeometric functions given in [7, 2.8(20)-(27)] and [18, § 15.5]. The first fractional generalization of one of these differentiation formulas was given by Bateman [4, p. 184]. Fractional generalizations of some further differentiation formulas were given by Askey \& Fitch [3, Section 2]. One still missing case was partially handled by Camporesi [5, paragraph after (2.28)]. In this paper the full list of the eight fractional integral transformation formulas will be given.

Another observation leading to this paper was that Euler's integral representation for the Gauss hypergeometric function, when written as a fractional integral

$$
\begin{aligned}
{ }_{2} F_{1}\left(\begin{array}{c}
a, b \\
c
\end{array} ; x\right)= & \frac{\Gamma(c)}{\Gamma(b) \Gamma(c-b)} x^{1-c} \int_{0}^{x} y^{b-1}(1-y)^{-a}(x-y)^{c-b-1} d y \\
& (0<x<1, \operatorname{Re} c>\operatorname{Re} b>0),
\end{aligned}
$$

should have a proof by using the hypergeometric differential equation [1, (2.3.5)]

$$
L_{a, b, c ; z}\left({ }_{2} F_{1}\left(\begin{array}{c}
a, b \\
c
\end{array} ; z\right)\right)=0
$$

${ }^{\star}$ This paper is a contribution to the Special Issue on Exact Solvability and Symmetry Avatars in honour of Luc Vinet. The full collection is available at http://www.emis.de/journals/SIGMA/ESSA2014.html 
where

$$
L_{a, b, c ; z}(f(z))=\left(L_{a, b, c} f\right)(z):=z(1-z) f^{\prime \prime}(z)+(c-(a+b+1) z) f^{\prime}(z)-a b f(z),
$$

and that then essentially the same proof should also yield that

$$
f(x):=|x|^{1-c} \int_{m}^{M}|y|^{b-1}|1-y|^{-a}|x-y|^{c-b-1} d y
$$

is a solution of the hypergeometric differential equation if $m, M$ and $x$ are as follows:

\begin{tabular}{|r||c|c|c|c|c|c|c|c|c|c|}
\hline$(m, M)$ & $(-\infty, x)$ & $(-\infty, 0)$ & $(x, 0)$ & $(0, x)$ & $(0,1)$ & $(0,1)$ & $(x, 1)$ & $(1, x)$ & $(1, \infty)$ & $(x, \infty)$ \\
\hline$x \in$ & $(-\infty, 0)$ & $(0, \infty)$ & $(-\infty, 0)$ & $(0,1)$ & $(-\infty, 0)$ & $(1, \infty)$ & $(0,1)$ & $(1, \infty)$ & $(-\infty, 1)$ & $(1, \infty)$ \\
\hline
\end{tabular}

This means that $m$ and $M$ in (1.4) are two consecutive points of singularity of the integrand. Indeed, we will see that in all listed cases the right-hand side of (1.4) equals a constant multiple of one of the six explicit solutions $w_{j}[18, \S 15.10(\mathrm{ii})]$ of the hypergeometric differential equation. In fact, as was Sergei Sitnik kindly commenting to me following an earlier version of this paper, the above observations (in the fractional integral case where $m$ or $M$ equals $x$ ) were already made in great detail by Letnikov [14] in 1874 in a paper which seems to have been unobserved outside Russia.

The Euler type integral representations of fractional integral type are specializations of parameter changing fractional integral transforms acting on some $w_{j}$. For instance, (1.1) is a special case of Bateman's fractional integral formula [4, p. 184]:

$$
\int_{0}^{x} y_{2}^{c-1} F_{1}\left(\begin{array}{c}
a, b \\
c
\end{array} ; y\right) \frac{(x-y)^{\mu-1}}{\Gamma(\mu)} d y=\frac{\Gamma(c)}{\Gamma(c+\mu)} x_{2}^{c+\mu-1} F_{1}\left(\begin{array}{c}
a, b \\
c+\mu
\end{array} ; x\right)
$$

$$
(0<x<1, \operatorname{Re} c>0, \operatorname{Re} \mu>0) \text {. }
$$

It will turn out that (1.5), and all other fractional integral formulas for hypergeometric functions to be considered, admit a proof by using the hypergeometric differential equation. Here $L_{a, b, c}$, given by (1.3), occurs in so-called transmutation formulas, for instance in connection with (1.5):

$$
\begin{gathered}
L_{a, b, c+\mu ; x}\left(x^{1-c-\mu} \int_{0}^{x} y^{c-1} f(y) \frac{(x-y)^{\mu-1}}{\Gamma(\mu)} d y\right)=x^{1-c-\mu} \int_{0}^{x} y^{c-1}\left(L_{a, b, c} f\right)(y) \frac{(x-y)^{\mu-1}}{\Gamma(\mu)} d y \\
\left(f \in C^{2}([0,1)), x \in(0,1), \operatorname{Re} c>0, \operatorname{Re} \mu>0\right) .
\end{gathered}
$$

Transmutation is a term which occurs in many meanings in science, and even can have various meanings in mathematics, but in the sense used here it was first considered in great detail by Lions [15], namely as an operator $A$, often an integral operator, intertwining between two differential operators $L_{1}$ and $L_{2}$ :

$$
L_{1} A=A L_{2} .
$$

In most examples, but not here, $L_{1}=d^{2} / d x^{2}$. Then a typical example for $L_{2}$ would be the Bessel type differential operator $L_{2}=d^{2} / d x^{2}+a x^{-1} d / d x$. Lions [15] built on earlier work by J. Delsarte (1938) and Levitan (1951). Many papers and books on transmutation have appeared since then. See the surveys $[21,22]$ by Sitnik and references given there.

The cases of (1.4) where $m$ and $M$ are not equal to $x$ are variants of the generalized Stieltjes transform, introduced by Widder [26, Section 8], and further developed by many authors, see for instance references in $[10,11]$. We consider the generalized Stieltjes transform as a transform sending $f$ to $g$ of the form

$$
g(x)=\int_{m}^{M} f(y)|y-x|^{\mu-1} d y,
$$


where $(m, M)$ is $(-\infty, 0)$ or $(0,1)$ or $(1, \infty)$, where $x$ is on $\mathbb{R}$ outside the integration interval, and where the function $y \mapsto|y|^{\mu-1} f(y)$ is $L^{1}$ on the integration interval. The Euler type integral representations of this form are special cases of generalized Stieltjes transforms which send a solution $w_{i}$ of the hypergeometric differential equation to a solution $w_{j}$ and change the parameters.

Some of these formulas can be found in literature, notably in the Bateman project [8]. There $[8,14.4(9)]$ is essentially the case $(m, M)=(-\infty, 0)$ of $(1.4)$, while $[8,20.2(10)]$ is a generalized Stieltjes transform sending a ${ }_{2} F_{1}$ to a ${ }_{2} F_{1}$. A formula by Karp \& Sitnik [12, Lemma 2] is essentially a generalized Stieltjes transform sending a ${ }_{2} F_{1}$ to a ${ }_{3} F_{2}$, which can be specialized to a transform sending ${ }_{2} F_{1}$ to ${ }_{2} F_{1}$. We will give a long list of generalized Stieltjes transforms sending some $w_{i}$ to some $w_{j}$, including the two from literature just mentioned. The formulas in this list are essentially equivalent: they can all be derived from each other.

The case $\mu=0$ of (1.7) is essentially the classical Stieltjes transform. It will not change the parameters of the hypergeometric solutions. A well-known example of this case is [25, (4.61.4)], which sends Jacobi polynomials to Jacobi functions of the second kind.

The idea that formulas for hypergeometric functions can be proved by using the hypergeometric differential equation goes back to Riemann. See Andrews, Askey \& Roy [1, Sections 2.3 and 3.9] how this method can be used for a proof of Pfaff's and Euler's transformation formulas and of quadratic transformation formulas. Such methods are recently also used by Paris and coauthors $[13,24]$. They refer to an earlier proof in this spirit by Rainville [19, p. 126] of a quadratic transformation formula involving a ${ }_{1} F_{1}$ and a ${ }_{0} F_{1}$. In this paper the method will be applied to integral formulas, but with a different focus.

Our message is that many formulas for hypergeometric functions have a companion formula involving the hypergeometric differential equation, which is more universal because it will imply or suggest many formulas involving the various solutions $w_{j}$ of the hypergeometric differential equation. A final rigorous proof of these formulas may not use the universal formula (as will be often the case in the present paper), but the universal formula is helpful for arriving at these formulas and for organizing them.

Quite probably the ideas of this paper will also work in other situations, for instance for Appell hypergeometric series.

The contents of this paper are as follows. After some preliminaries for hypergeometric functions in Section 2, we illustrate in Section 3 the main ideas of the paper for the special case of the Bateman integral (1.5). This takes quite a few pages, but it is less technical than the rest of the paper. In Section 4 we state and prove the eight fractional integral transformations corresponding to the eight $n$-th derivative formulas for hypergeometric functions. We give also eight corresponding transmutation formulas. In Section 5 we discuss the 48 fractional integration formulas for the six solutions $w_{j}$, which can be obtained by rewriting the formulas in Section 4 . Not all formulas will be given explicitly. In Section 6 we give 24 generalized Stieltjes transforms sending some $w_{i}$ to some $w_{j}$. In Section 7 we give for each of the six solutions $w_{j}$ two Euler type integral representations. They can all be obtained by specialization of formulas in Sections 5 and 6. Finally, Section 8 discusses the connection between generalized Stieltjes transforms of different order by fractional integration, and how this leads to connections between formulas in Sections 5 and 6 .

\section{Preliminaries about hypergeometric functions}

The Gauss hypergeometric function [7, Chapter 2], [1, Chapter 2], [18, Chapter 15] is defined by its power series

$$
{ }_{2} F_{1}\left(\begin{array}{c}
a, b \\
c
\end{array} ; z\right)=F(a, b ; c ; z):=\sum_{k=0}^{\infty} \frac{(a)_{k}(b)_{k}}{(c)_{k} k !} z^{k} \quad(|z|<1) .
$$


Here the (complex) parameters $a, b, c$ are taken generically. In particular the series should be well-defined $\left(c \notin \mathbb{Z}_{\leq 0}\right.$ ), while possibly nicer results in case of terminating series $\left(a\right.$ or $\left.b \in \mathbb{Z}_{\leq 0}\right)$ are not paid attention to. The function (2.1) has a one-valued analytic continuation to $\mathbb{C} \backslash[1, \infty)$. It satisfies the hypergeometric differential equation (1.2) and it is uniquely determined as the function regular near 0 and equal to 1 at 0 which is annihilated by $L_{a, b, c}$.

Important transformation formulas, due to Pfaff and Euler, respectively, are [1, (2.2.6), $(2.2 .7)],[18,(15.8 .1)]$ :

$$
\begin{aligned}
& { }_{2} F_{1}\left(\begin{array}{c}
a, b \\
c
\end{array} ; z\right)=(1-z)^{-a}{ }_{2} F_{1}\left(\begin{array}{c}
a, c-b \\
c
\end{array} \frac{z}{z-1}\right) \quad(z \notin[1, \infty)), \\
& { }_{2} F_{1}\left(\begin{array}{c}
a, b \\
c
\end{array} ; z\right)=(1-z)^{c-a-b}{ }_{2} F_{1}\left(\begin{array}{c}
c-a, c-b \\
c
\end{array} ; z\right) \quad(z \notin[1, \infty)) .
\end{aligned}
$$

Often, a specific formula for $F(a, b ; c ;$.$) trivially implies another one by the symmetry in a$ and $b$. For instance, when we will refer to (2.2), we may also mean a similar identity with the right-hand side given by $(1-z)^{-b} F(c-a, b ; c ; z /(z-1))$. An elementary special case of the hypergeometric function is

$$
{ }_{2} F_{1}\left(\begin{array}{c}
a, b \\
b
\end{array} ; z\right)=(1-z)^{-a} \quad(z \notin[1, \infty))
$$

In the case of generic parameters there are essentially six different explicit solutions of the Gauss differential equation $L_{a, b, c} f=0$ [7, Section 2.9], [18, $\left.\S 15.10(i i)\right]$. These are one-valued analytic functions on the complex plane with a suitable real interval as cut:

$$
\begin{array}{ll}
w_{1}(z ; a, b, c):={ }_{2} F_{1}\left(\begin{array}{c}
a, b \\
c
\end{array} z\right) & (z \notin[1, \infty)), \\
w_{2}(z ; a, b, c):=z^{1-c}{ }_{2} F_{1}\left(\begin{array}{c}
a-c+1, b-c+1 \\
2-c
\end{array} ;\right) & (z \notin(-\infty, 0] \cup[1, \infty)), \\
w_{3}(z ; a, b, c):=z^{-a}{ }_{2} F_{1}\left(\begin{array}{c}
a, a-c+1 \\
a-b+1
\end{array} ; z^{-1}\right) & (z \notin(-\infty, 1]), \\
w_{4}(z ; a, b, c):=z^{-b}{ }_{2} F_{1}\left(\begin{array}{c}
b, b-c+1 \\
b-a+1
\end{array} ; z^{-1}\right) & (z \notin(-\infty, 1]), \\
w_{5}(z ; a, b, c):={ }_{2} F_{1}\left(\begin{array}{c}
a, b \\
a+b-c+1
\end{array} ; 1-z\right) & (z \notin(-\infty, 0]), \\
w_{6}(z ; a, b, c):=(1-z)^{c-a-b}{ }_{2} F_{1}\left(\begin{array}{c}
c-a, c-b \\
c-a-b+1
\end{array} ; 1-z\right) & (z \notin(-\infty, 0] \cup[1, \infty)) .
\end{array}
$$

Here we had to exclude not just the branch cuts of the ${ }_{2} F_{1}$ 's, but also those of the power factors (we assume principal values for the complex powers). In the case of $w_{2}, w_{3}, w_{4}, w_{6}$ we might have chosen the branch cuts due to the power factors differently. For instance, a companion of $w_{2}$ would be

$$
\widetilde{w}_{2}(z ; a, b, c):=(-z)^{1-c}{ }_{2} F_{1}\left(\begin{array}{c}
a-c+1, b-c+1 \\
2-c
\end{array} ; z\right) \quad(z \notin[0, \infty)) .
$$

We will also consider the six solutions on subintervals of the real axis including the branch cuts of the power factors as below (here we abuse notation by not changing it compared to 
above)

$$
\begin{array}{ll}
w_{1}(x ; a, b, c)={ }_{2} F_{1}\left(\begin{array}{c}
a, b \\
c
\end{array} ;\right) & (x \in(-\infty, 1)), \\
w_{2}(x ; a, b, c)=|x|^{1-c}{ }_{2} F_{1}\left(\begin{array}{c}
a-c+1, b-c+1 \\
2-c
\end{array} ; x\right) & (x \in(-\infty, 0) \cup(0,1)), \\
w_{3}(x ; a, b, c)=|x|^{-a}{ }_{2} F_{1}\left(\begin{array}{c}
a, a-c+1 \\
a-b+1
\end{array} ; x^{-1}\right) & (x \in(-\infty, 0) \cup(1, \infty)), \\
w_{4}(x ; a, b, c)=|x|^{-b}{ }_{2} F_{1}\left(\begin{array}{c}
b, b-c+1 \\
b-a+1
\end{array} x^{-1}\right) & (x \in(-\infty, 0) \cup(1, \infty)), \\
w_{5}(x ; a, b, c)={ }_{2} F_{1}\left(\begin{array}{c}
a, b \\
a+b-c+1
\end{array} ;-x\right) & (x \in(0, \infty)), \\
w_{6}(x ; a, b, c)=|1-x|^{c-a-b}{ }_{2} F_{1}\left(\begin{array}{c}
c-a, c-b \\
c-a-b+1
\end{array} ; 1-x\right) & (x \in(0,1) \cup(1, \infty)) .
\end{array}
$$

\section{The main ideas illustrated in a special case}

\subsection{Transmutation property of a differentiation operator}

In $[7,2.8(20)-(27)]$ or $[18, \S 15.5]$ there is a list of eight parameter changing $n$-th derivative formulas for Gauss hypergeometric functions. One of these is [18, (15.5.4)]:

$$
\left(\frac{d}{d z}\right)^{n}\left(z_{2-1}^{c-1} F_{1}\left(\begin{array}{c}
a, b \\
c
\end{array} ; z\right)\right)=\frac{\Gamma(c)}{\Gamma(c-n)} z^{c-n-1}{ }_{2} F_{1}\left(\begin{array}{c}
a, b \\
c-n
\end{array} ; z\right) \text {. }
$$

Formula (3.1) can be proved immediately by power series expansion (2.1). Note also that the $n$-th derivative case follows by iteration of the case $n=1$. The case $n=1$ can be rewritten as

$$
D_{c-12} F_{1}\left(\begin{array}{c}
a, b \\
c
\end{array} ; \cdot\right)=(c-1){ }_{2} F_{1}\left(\begin{array}{c}
a, b \\
c-1
\end{array} ; \cdot\right),
$$

where

$$
\left(D_{a} f\right)(z):=z f^{\prime}(z)+a f(z)=z^{-a+1} \frac{d}{d z}\left(z^{a} f(z)\right) .
$$

Clearly, if $f$ is analytic at 0 then $D_{a} f$ is analytic at 0 and $\left(D_{a} f\right)(0)=a f(0)$.

Straightforward computation followed by iteration gives the following transmutation property:

$$
\begin{aligned}
& L_{a, b, c-1} D_{c-1}=D_{c-1} L_{a, b, c} \\
& L_{a, b, c-n} D_{c-n} \cdots D_{c-2} D_{c-1}=D_{c-n} \cdots D_{c-2} D_{c-1} L_{a, b, c} .
\end{aligned}
$$

Formula (3.2) is also a consequence of (3.4). Indeed, by (3.4) $L_{a, b, c-1}$ annihilates the left-hand side of (3.2). Since this left-hand side is regular at 0 , it must be a constant times $F(a, b ; c-1 ;$. with the constant obtained by evaluating both sides at 0. Similarly, (3.1) is a consequence of (3.5).

Because of the above argument, it is natural to consider $D_{c-1} w_{j}(. ; a, b, c)$ not just for $j=1$ but also for the other explicit solutions of the hypergeometric differential equation $(j=2, \ldots, 6)$. It will turn out that for all $j$ we get some constant factor times $w_{j}(. ; a, b, c-1)$. For instance, with $w_{2}$ given by $(2.6)$,

$$
D_{c-1} w_{2}(. ; a, b, c)=\frac{(a-c+1)(b-c+1)}{2-c} w_{2}(. ; a, b, c-1) .
$$


This is equivalent to the prototypical differentiation formula for the hypergeometric function [4, (15.5.4)]:

$$
\frac{d}{d z}{ }_{2} F_{1}\left(\begin{array}{c}
a, b \\
c
\end{array} ; z\right)=\frac{a b}{c}{ }_{2} F_{1}\left(\begin{array}{c}
a+1, b+1 \\
c+1
\end{array} ; z\right)
$$

Corresponding to each of the eight $n$-th derivative formulas [18, (15.5.2)-(15.5.9)] one can write down a transmutation formula like (3.5), and corresponding to each of these transmutation formulas one can write down an $n$-th derivative formula for each of the six solutions $w_{j}$. These will not be included in the present paper. The transmutation formulas for the first derivative operators were earlier given by Dereziński [6, end of Section 3.4]. He calls them commutation relations and he relates them to to the Lie algebra $\mathfrak{s o ( 6 )}$ (or equivalently $\mathfrak{s l}(4)$ ). This way to associate a Lie algebra (the so-called dynamical symmetry algebra) with a hypergeometric differential equation was first described by Miller [17]. This was later understood in the framework of $\mathcal{A}$-hypergeometric systems, see Saito [20].

Remark 3.1. The transformation formulas (2.2) and (2.3) also have a more universal background because they can be understood from transformation properties of the differential operator $L_{a, b, c}$ (see also $[1,(2.3 .10 \mathrm{~B})$ and $\left.(2.3 .10 \mathrm{~F})]\right)$ :

$$
\begin{aligned}
& L_{a, b, c ; z}\left((1-z)^{-a} f\left(\frac{z}{z-1}\right)\right)=-(1-z)^{-a-1}\left(L_{a, c-b, c} f\right)\left(\frac{z}{z-1}\right), \\
& L_{a, b, c ; z}\left((1-z)^{c-a-b} f(z)\right)=(1-z)^{c-a-b}\left(L_{c-a, c-b, c} f\right)(z) .
\end{aligned}
$$

This suggests identities involving $w_{i}(z ; a, b, c),(1-z)^{-a} w_{j}\left(\frac{z}{z-1} ; a, c-b, c\right),(1-z)^{-b} w_{k}\left(\frac{z}{z-1}\right.$; $c-a, b, c)$ and $(1-z)^{c-a-b} w_{l}(z ; c-a, c-b, c)$. These can indeed be given, but sometimes one needs another branch cut for the power factor than chosen in (2.5)-(2.10).

\subsection{Transmutation property of a fractional integral operator}

Both Riemann-Liouville and Weyl fractional integrals will occur in our formulas. See, for instance, [8, pp. 181-183] or [16].

Formula (3.1) can equivalently be written as a repeated integral, which can be condensed to a single integral [1, p. 111]. For this purpose, take $0<x<1$ and assume $\operatorname{Re} c>n$. We obtain

$$
x^{c-1}{ }_{2} F_{1}\left(\begin{array}{c}
a, b \\
c
\end{array} ; x\right)=\frac{\Gamma(c)}{\Gamma(c-n)} \int_{0}^{x} y^{c-n-1}{ }_{2} F_{1}\left(\begin{array}{c}
a, b \\
c-n
\end{array} ; y\right) \frac{(x-y)^{n-1}}{(n-1) !} d y .
$$

Note that there are no other terms because, by our assumption, $y^{c-m}$ vanishes at $y=0$ for $m=1, \ldots, n$. Formula (3.10) has a fractional extension for $n$ complex with $\operatorname{Re} c>\operatorname{Re} n>0$. In rewritten form this is Bateman's fractional integral formula (1.5), which is often written in the form

$$
\begin{aligned}
\int_{0}^{1} t^{c-1}{ }_{2} F_{1}\left(\begin{array}{c}
a, b \\
c
\end{array} ; t z\right) \frac{(1-t)^{\mu-1}}{\Gamma(\mu)} d t= & \frac{\Gamma(c)}{\Gamma(c+\mu)}{ }_{2} F_{1}\left(\begin{array}{c}
a, b \\
c+\mu
\end{array} ; z\right) \\
& (z \in \mathbb{C} \backslash[1, \infty), \operatorname{Re} c>0, \operatorname{Re} \mu>0) .
\end{aligned}
$$

Then (1.5) follows from (3.11) by specializing $z$ to $x \in(0,1)$ and substituting $t=y / x$. By analytic continuation it is sufficient to prove (3.11) for $|z|<1$. There it follows by power series expansion (2.1). For (3.11) see also [3, (2.4)], [2, (3.5)] and [1, Theorem 2.2.4, (2.9.6)].

Just as we proved (3.2) by (3.4), we can prove (1.5) from the following identity (derived by straightforward computation):

$$
y^{1-c} L_{1-a, 1-b, 2-c ; y}\left(y^{c-1}(x-y)^{\mu-1}\right)=x^{c+\mu-1} L_{a, b, c+\mu ; x}\left(x^{1-c-\mu}(x-y)^{\mu-1}\right) .
$$

We also need (straightforward by integration by parts): 
Lemma 3.2. Let $f, g \in C^{2}((m, M))$. Then, for $x \in(m, M)$,

$$
\begin{aligned}
& \left(L_{a, b, c} f\right)(x) g(x)-f(x)\left(L_{1-a, 1-b, 2-c} g\right)(x) \\
& \quad=\frac{d}{d x}\left(x(1-x)\left(f^{\prime}(x) g(x)-f(x) g^{\prime}(x)\right)+(c-1+(1-a-b) x) f(x) g(x)\right) .
\end{aligned}
$$

Furthermore, let $x(1-x) f^{\prime \prime}(x) g(x), x(1-x) f(x) g^{\prime \prime}(x),(1+|x|) f^{\prime}(x) g(x),(1+|x|) f(x) g^{\prime}(x)$, $f(x) g(x)$, as functions of $x$, be in $L^{1}((m, M))$, and assume that

$$
\left.\begin{array}{l}
x(1-x) f^{\prime}(x) g(x) \\
x(1-x) f(x) g^{\prime}(x) \\
(1+|x|) f(x) g(x)
\end{array}\right\} \rightarrow 0 \quad \text { as } x \downarrow m \text { or } x \uparrow M .
$$

Then

$$
\int_{m}^{M}\left(L_{a, b, c} f\right)(x) g(x) d x=\int_{m}^{M} f(x)\left(L_{1-a, 1-b, 2-c} g\right)(x) d x .
$$

In particular, $L_{1-a, 1-b, 2-c}$ is the formal adjoint of $L_{a, b, c}$.

Proof of (1.5) by (3.12). First assume $0<x<1, \operatorname{Re} c>1$, $\operatorname{Re} \mu>2$, We have

$$
\begin{aligned}
& L_{a, b, c+\mu ; x}\left(x^{1-c-\mu} \times \text { left-hand side of }(1.5)\right) \\
& =\frac{1}{\Gamma(\mu)} \int_{0}^{x} y^{c-1}{ }_{2} F_{1}\left(\begin{array}{c}
a, b \\
c
\end{array} ; y\right) L_{a, b, c+\mu ; x}\left(x^{1-c-\mu}(x-y)^{\mu-1}\right) d y \\
& =\frac{x^{1-c-\mu}}{\Gamma(\mu)} \int_{0}^{x}{ }_{2} F_{1}\left(\begin{array}{c}
a, b \\
c
\end{array} ; y\right) L_{1-a, 1-b, 2-c ; y}\left(y^{c-1}(x-y)^{\mu-1}\right) d y \\
& =\frac{x^{1-c-\mu}}{\Gamma(\mu)} \int_{0}^{x} L_{a, b, c ; y}\left({ }_{2} F_{1}\left(\begin{array}{c}
a, b \\
c
\end{array} ; y\right)\right) y^{c-1}(x-y)^{\mu-1} d y=0 .
\end{aligned}
$$

For the first equality sign we twice used a generalized Leibniz rule [9, (1.1)] (allowed because the integral in the second line converges absolutely) together with the vanishing of the integrand of the left-hand side of (1.5) for $y=x$ (and similarly for the derivative with respect to $x$ of that integrand $)^{1}$. For the second equality apply (3.12) and for the third equality (3.13) (the conditions are satisfied). Because

$$
x^{1-c-\mu} \times \text { left-hand side of }(1.5)=\int_{0}^{1} t^{c-1}{ }_{2} F_{1}\left(\begin{array}{c}
a, b \\
c
\end{array} t x\right) \frac{(1-t)^{\mu-1}}{\Gamma(\mu)} d t,
$$

this is analytic in $x$ at $x=0$, and for $x=0$ it takes the value

$$
\int_{0}^{1} t^{c-1} \frac{(1-t)^{\mu-1}}{\Gamma(\mu)} d t=\frac{\Gamma(c)}{\Gamma(c+\mu)} .
$$

Thus, by the characterization of the hypergeometric function as solution of the hypergeometric differential equation, we have proved (3.12) for $\operatorname{Re} \mu>2$, $\operatorname{Re} c>1$. These conditions can be relaxed by analytic continuation.

If we replace in the above proof the ${ }_{2} F_{1}$ by a suitable function $f$ then we obtain the transmutation property (1.6).

\footnotetext{
${ }^{1}$ The involved differentiation under the integral sign got some fame as "Feynman's trick", see https://en. wikipedia.org/wiki/Differentiation_under_the_integral_sign\#Popular_culture.
} 
Remark 3.3. For $\operatorname{Re} \mu>2$ (1.6) is equivalent to (3.12). For $\mu=3,4, \ldots$ (1.6) is also equivalent to (3.5). Furthermore, it is sufficient to prove (3.12) for $\mu=3,4, \ldots$ because, after division of both sides by $(x-y)^{\mu-1}$, the two sides depend polynomially on $\mu$.

Formally, the proof of (1.6) can be extended to showing that

$$
\begin{aligned}
L_{a, b, c+\mu ; x}\left(|x|^{1-c-\mu} \int_{m}^{M}|y|^{c-1} f(y) \frac{|x-y|^{\mu-1}}{\Gamma(\mu)} d y\right) \\
=|x|^{1-c-\mu} \int_{m}^{M}|y|^{c-1}\left(L_{a, b, c} f\right)(y) \frac{|x-y|^{\mu-1}}{\Gamma(\mu)} d y .
\end{aligned}
$$

Here $m$ or $M$ may be equal to $x$, but not necessarily, and $y$ and $x-y$ should not change sign for $y \in[m, M)$. This becomes rigorous if $f, c$ and $\mu$ are such that the first and third equality (suitably modified) in the above Proof remain valid in the case of (3.14). In particular, $f$ should have suitable vanishing properties at $m$ and $M$, even stronger if $m$ or $M$ are not equal to 0 or $x$.

If (3.14) holds and if $L_{a, b, c} f=0$ then

$$
L_{a, b, c+\mu} g=0, \quad g(x):=|x|^{1-c-\mu} \int_{m}^{M}|y|^{c-1} f(y) \frac{|x-y|^{\mu-1}}{\Gamma(\mu)} d y .
$$

In this paper many explicit cases of (3.15) will be given, where $f=w_{i}$ for some $i$ and $g=$ const. $w_{j}$ for some $j$, with $j=i$ if $m$ or $M$ equals $x$. As an example it can be derived that

$$
\begin{aligned}
& \int_{-\infty}^{x}(-y)^{c-1} w_{2}(y ; a, b, c) \frac{(x-y)^{\mu-1}}{\Gamma(\mu)} d y= \frac{\Gamma(a-c-\mu+1)}{\Gamma(a-c+1)} \frac{\Gamma(b-c-\mu+1)}{\Gamma(b-c+1)} \frac{\Gamma(2-c)}{\Gamma(2-c-\mu)} \\
& \times(-x)^{c+\mu-1} w_{2}(x ; a, b, c+\mu) \\
&(x<0, \operatorname{Re}(a-c-\mu+1), \operatorname{Re}(b-c-\mu+1), \operatorname{Re} \mu>0) .
\end{aligned}
$$

This is the fractional generalization of the iteration of (3.6). It can be equivalently written as

$$
\begin{gathered}
\int_{-\infty}^{x}{ }_{2} F_{1}\left(\begin{array}{c}
a, b \\
c
\end{array} ; y\right) \frac{(x-y)^{\mu-1}}{\Gamma(\mu)} d y=\frac{\Gamma(a-\mu)}{\Gamma(a)} \frac{\Gamma(b-\mu)}{\Gamma(b)} \frac{\Gamma(c)}{\Gamma(c-\mu)}{ }_{2} F_{1}\left(\begin{array}{c}
a-\mu, b-\mu \\
c-\mu
\end{array} ; x\right) \\
(x<0, \operatorname{Re} a, \operatorname{Re} b>\operatorname{Re} \mu>0) .
\end{gathered}
$$

This is the fractional generalization of the iteration of (3.7). A different proof will be given in the next section.

Another explicit case of (3.15) which we will meet is

$$
\begin{aligned}
\int_{1}^{\infty} y^{c-1} w_{6}(y ; a, b, c)(y-x)^{\mu-1} d y \\
=\frac{\Gamma(a-c-\mu+1) \Gamma(b-c-\mu+1) \Gamma(c-a-b+1)}{\Gamma(2-c-\mu) \Gamma(1-\mu)} x^{c+\mu-1} w_{2}(x ; a, b, c+\mu) \\
\quad(0<x<1, \operatorname{Re}(a-c-\mu+1), \operatorname{Re}(b-c-\mu+1), \operatorname{Re}(c-a-b+1)>0) .
\end{aligned}
$$

The left-hand side is no longer of fractional integral type, but it is a generalized Stieltjes transform, to which we will return in a moment.

\subsection{Euler type integral representations}

When we replace $c, \mu$ by $b, c-b$ in (1.5) or (3.11) and use (2.4) then we obtain Euler's integral representation as fractional integral (1.1), or in its most used form

$$
\begin{aligned}
{ }_{2} F_{1}\left(\begin{array}{c}
a, b \\
c
\end{array} ; z\right)= & \frac{\Gamma(c)}{\Gamma(b) \Gamma(c-b)} \int_{0}^{1} t^{b-1}(1-t)^{c-b-1}(1-t z)^{-a} d t \\
& (z \in \mathbb{C} \backslash[1, \infty), \operatorname{Re} c>\operatorname{Re} b>0) .
\end{aligned}
$$


The right-hand side of (1.1) is annihilated by $L_{a, b, c ; x}$. This is a consequence of the transmutation property (1.6), by which $L_{a, b, c ; x}$ acting on the right-hand side of (1.1) is equal to

$$
\text { const } \cdot x^{1-c} \int_{0}^{x} y^{b-1} L_{a, b, b ; y}\left((1-y)^{-a}\right)(x-y)^{c-b-1} d y=0
$$

because $(1-y)^{-a}$ is annihilated by $L_{a, b, b ; y}$. This last fact is also apparent from

$$
L_{a, b, b ; y}=\left(y \frac{d}{d y}+b\right) \circ\left((1-y) \frac{d}{d y}-a\right) .
$$

The more general transmutation property (3.14), considered with $c, \mu$ replaced by $b, c-b$, suggests that each $w_{j}$ has an Euler type integral representation (1.4). This is indeed the case, as we already briefly indicated after (1.4).

We can write (3.19) also equivalently as a generalized Stieltjes transform

$$
\begin{aligned}
w_{3}(x ; a, b, c)= & \frac{\Gamma(a-b+1)}{\Gamma(a-c+1) \Gamma(c-b)} \int_{0}^{1} y^{a-c}(1-y)^{c-b-1}(x-y)^{-a} d y \\
& (x>1, \operatorname{Re}(a+1)>\operatorname{Re} c>\operatorname{Re} b) .
\end{aligned}
$$

It will turn out that, more generally and analogous to (1.4),

$$
g(x)=\int_{m}^{M}|y|^{a-c}|1-y|^{c-b-1}|x-y|^{-a} d y
$$

is a solution of the hypergeometric differential equation if $m, M$ and $x$ are as listed after (1.4).

Although most Euler type integral representations of fractional integral type are equivalent to some integral representation of generalized Stieltjes transform type by a change of integration variable, this is no longer true for the integral transforms mapping $w_{i}$ to $w_{j}$ which specialize to an Euler type integral representation.

\subsection{Generalized Stieltjes transforms as transmutation operators}

We already observed in Section 1 that variants of fractional integral transforms and the Euler integral representation for hypergeometric functions naturally lead to formulas involving a generalized Stieltjes transform. In the definition by Widder [26, Section 8] the generalized Stieltjes transform sends a suitable measure $\alpha$ or function $\phi$ (with $d \alpha(t)=\phi(t) d t$ ) to a function $f$ analytic on $\mathbb{C} \backslash(-\infty, 0]$ :

$$
\int_{0}^{\infty} \frac{d \alpha(t)}{(z+t)^{\rho}}=f(z)
$$

The special case $\rho=1$ gives the classical Stieltjes transform. In order to have analytic expressions similar to the ones in fractional integral transforms, we will work with transforms (1.7).

Transforms of generalized Stieltjes type have transmutation properties. For instance, from (3.14) we have, associated with (3.18), the intertwining property

$$
\begin{aligned}
& L_{a, b, c+\mu ; x}\left(x^{1-c-\mu} \int_{1}^{\infty} y^{c-1} f(y)(y-x)^{\mu-1} d y\right) \\
& \quad=x^{1-c-\mu} \int_{1}^{\infty} y^{c-1}\left(L_{a, b, c} f\right)(y)(y-x)^{\mu-1} d y \quad(0<x<1) .
\end{aligned}
$$

Noteworthy is the case $\mu=0$. Then we have the same hypergeometric differential operator on both sides. The Stieltjes transform will then map solutions of the differential equation to other solutions. 
Karp \& Sitnik [12, Lemma 2] proved the following formula:

$$
\begin{gathered}
\int_{0}^{1} t^{b-1}(1-t)^{d+e-b-c-1}{ }_{2} F_{1}\left(\begin{array}{c}
d-c, e-c \\
d+e-b-c
\end{array} ; 1-t\right)(1-t z)^{-a} d t \\
=\frac{\Gamma(b) \Gamma(c) \Gamma(d+e-b-c)}{\Gamma(d) \Gamma(e)}{ }_{3} F_{2}\left(\begin{array}{c}
a, b, c \\
d, e
\end{array} ; z\right) \\
(z \in \mathbb{C} \backslash[1, \infty), \operatorname{Re} b, \operatorname{Re} c, \operatorname{Re}(d+e-b-c)>0) .
\end{gathered}
$$

If we replace $z$ by $z^{-1}$ then we recognize the formula as a generalized Stieltjes transform sending $\mathrm{a}_{2} F_{1}$ to a ${ }_{3} F_{2}$ of general parameters. (For more general formulas expressing ${ }_{p} F_{p-1}$ functions as generalized Stieltjes transforms see [11] and references given there.) If moreover $d=a$ the transform in [19] sends a ${ }_{2} F_{1}$ to a ${ }_{2} F_{1}$ :

$$
\begin{gathered}
\int_{0}^{1} t^{b-1}(1-t)^{a+e-b-c-1}{ }_{2} F_{1}\left(\begin{array}{c}
a-c, e-c \\
a+e-b-c
\end{array} ; 1-t\right)(z-t)^{-a} d t \\
=\frac{\Gamma(b) \Gamma(c) \Gamma(a+e-b-c)}{\Gamma(a) \Gamma(e)} z^{-a}{ }_{2} F_{1}\left(\begin{array}{c}
b, c \\
e
\end{array} ; z^{-1}\right) \\
(z \in \mathbb{C} \backslash(-\infty, 1], \operatorname{Re} b, \operatorname{Re} c, \operatorname{Re}(d+e-b-c)>0) .
\end{gathered}
$$

All generalized Stieltjes transforms mapping a solution $w_{i}$ to a solution $w_{j}$ we know can be obtained from (3.23) by change of parameters, change of integration variable, and application of $(2.2)$ and (2.3).

\section{The eight fractional integral transformations of the Gauss hypergeometric function}

Each of the eight $n$-th derivative formulas in $[18, \S 15.5]$ has a fractional generalization. Some of these are very well-known, but others were hardly known until now. They fall apart into three families. Within a family the formulas follow from each other by application of (2.2) or (2.3). We will use shorthand names for the eight cases of which the meaning will be obvious. The division of the cases into families and their correspondence with the $n$-th derivative formulas is as follows:

\begin{tabular}{|l||lll|ll|lll|}
\hline family & I & I & I & II & II & III & III & III \\
\hline case & $c+$ & $a+, c+$ & $a+, b+, c+$ & $a-$ & $a+$ & $a-, b-, c-$ & $a-, c-$ & $c-$ \\
\hline$[18, \S 15.5]$ & $(4)$ & $(8)$ & $(9)$ & $(3)$ & $(5)$ & $(1)$ & $(7)$ & $(6)$ \\
\hline
\end{tabular}

\subsection{Family I}

Case $\boldsymbol{c}+$. We already discussed Bateman's fractional integral formula (1.5). From (3.11) we get another variant of (1.5):

$$
\begin{aligned}
\int_{x}^{0}(-y)^{c-1}{ }_{2} F_{1}\left(\begin{array}{c}
a, b \\
c
\end{array} ; y\right) \frac{(y-x)^{\mu-1}}{\Gamma(\mu)} d y= & \frac{\Gamma(c)}{\Gamma(c+\mu)}(-x)^{c+\mu-1}{ }_{2} F_{1}\left(\begin{array}{c}
a, b \\
c+\mu
\end{array} ; x\right) \\
& (x<0, \operatorname{Re} c>0, \operatorname{Re} \mu>0),
\end{aligned}
$$

which we can write together with (1.5) in a unified way as:

$$
\begin{gathered}
\int_{0<y / x<1}|y|^{c-1}{ }_{2} F_{1}\left(\begin{array}{c}
a, b \\
c
\end{array} ; y\right) \frac{|x-y|^{\mu-1}}{\Gamma(\mu)} d y=\frac{\Gamma(c)}{\Gamma(c+\mu)}|x|^{c+\mu-1}{ }_{2} F_{1}\left(\begin{array}{c}
a, b \\
c+\mu
\end{array} ; x\right) \\
(x \in(-\infty, 0) \cup(0,1), \operatorname{Re} c>0, \operatorname{Re} \mu>0) .
\end{gathered}
$$


Case $\boldsymbol{a}+, \boldsymbol{c}+$. By using (2.2) in (4.1) we arrive at

$$
\begin{gathered}
\int_{0<y / x<1}|y|^{c-1}(1-y)^{b-c-\mu}{ }_{2} F_{1}\left(\begin{array}{c}
a, b \\
c
\end{array} ; y\right) \frac{|x-y|^{\mu-1}}{\Gamma(\mu)} d y \\
=\frac{\Gamma(c)}{\Gamma(c+\mu)}|x|^{c+\mu-1}(1-x)^{b-c}{ }_{2} F_{1}\left(\begin{array}{c}
a+\mu, b \\
c+\mu
\end{array} ; x\right) \\
(x \in(-\infty, 0) \cup(0,1), \operatorname{Re} c>0, \operatorname{Re} \mu>0) .
\end{gathered}
$$

Case $\boldsymbol{a}+, \boldsymbol{b}+, \boldsymbol{c}+$. By using (2.3) in (4.1) we arrive at

$$
\begin{gathered}
\int_{0<y / x<1}|y|^{c-1}(1-y)^{a+b-c}{ }_{2} F_{1}\left(\begin{array}{c}
a, b \\
c
\end{array} ; y\right) \frac{|x-y|^{\mu-1}}{\Gamma(\mu)} d y \\
=\frac{\Gamma(c)}{\Gamma(c+\mu)}|x|^{c+\mu-1}(1-x)^{a+b-c+\mu}{ }_{2} F_{1}\left(\begin{array}{c}
a+\mu, b+\mu \\
c+\mu
\end{array} ; x\right) \\
(x \in(-\infty, 0) \cup(0,1), \operatorname{Re} c>0, \operatorname{Re} \mu>0) .
\end{gathered}
$$

See also Askey \& Fitch [3, (2.11)].

\subsection{Family II}

Case $a-$. Askey \& Fitch $[3,(2.10)]$ give

$$
\begin{gathered}
\int_{0}^{1} t^{a-\mu-1}{ }_{2} F_{1}\left(\begin{array}{c}
a, b \\
c
\end{array} ; z t\right) \frac{(1-t)^{\mu-1}}{\Gamma(\mu)} d t=\frac{\Gamma(a-\mu)}{\Gamma(a)}{ }_{2} F_{1}\left(\begin{array}{c}
a-\mu, b \\
c
\end{array} ;\right) \\
(z \notin(1, \infty), \operatorname{Re} a>\operatorname{Re} \mu>0) .
\end{gathered}
$$

The proof is as for (3.11). For $|z|<1$ the formula follows by power series expansion (2.1), and next the general case follows by analytic continuation. Formula (4.4) implies the following fractional integral formula:

$$
\begin{gathered}
\int_{0<y / x<1}|y|^{a-\mu-1}{ }_{2} F_{1}\left(\begin{array}{c}
a, b \\
c
\end{array} ; y\right) \frac{|x-y|^{\mu-1}}{\Gamma(\mu)} d y=\frac{\Gamma(a-\mu)}{\Gamma(a)}|x|^{a-1}{ }_{2} F_{1}\left(\begin{array}{c}
a-\mu, b \\
c
\end{array} ; x\right) \\
(x \in(-\infty, 0) \cup(0,1), \operatorname{Re} a>\operatorname{Re} \mu>0) .
\end{gathered}
$$

Case $\boldsymbol{a}+$. By using (2.2) or (2.3) in (4.5) we arrive at

$$
\begin{gathered}
\int_{0<y / x<1}|y|^{c-a-\mu-1}(1-y)^{a+b-c}{ }_{2} F_{1}\left(\begin{array}{c}
a, b \\
c
\end{array} ; y\right) \frac{|x-y|^{\mu-1}}{\Gamma(\mu)} d y \\
=\frac{\Gamma(c-a-\mu)}{\Gamma(c-a)}|x|^{c-a-1}(1-x)^{a+b-c+\mu}{ }_{2} F_{1}\left(\begin{array}{c}
a+\mu, b \\
c
\end{array} ; x\right) \\
(x \in(-\infty, 0) \cup(0,1), \operatorname{Re}(c-a)>\operatorname{Re} \mu>0) .
\end{gathered}
$$

\subsection{Family III}

Case $\boldsymbol{a}-, \boldsymbol{b}-, \boldsymbol{c}-$. The following generalizes a formula of Camporesi [5, paragraph after (2.28)].

Proposition 4.1. We have

$$
\begin{gathered}
\int_{-\infty}^{x}{ }_{2} F_{1}\left(\begin{array}{c}
a, b \\
c
\end{array} ; y\right) \frac{(x-y)^{\mu-1}}{\Gamma(\mu)} d y=\frac{\Gamma(a-\mu)}{\Gamma(a)} \frac{\Gamma(b-\mu)}{\Gamma(b)} \frac{\Gamma(c)}{\Gamma(c-\mu)}{ }_{2} F_{1}\left(\begin{array}{c}
a-\mu, b-\mu \\
c-\mu
\end{array} ; x\right) \\
(x<1, \operatorname{Re} a, \operatorname{Re} b>\operatorname{Re} \mu>0) .
\end{gathered}
$$


Proof. Formula (4.5) can be rewritten as:

$$
\begin{gathered}
\int_{1<\frac{y}{x}}|y|_{2}^{-a} F_{1}\left(\begin{array}{c}
a, b \\
c
\end{array} ; y^{-1}\right) \frac{|x-y|^{\mu-1}}{\Gamma(\mu)} d y=\frac{\Gamma(a-\mu)}{\Gamma(a)}|x|_{2}^{-a+\mu} F_{1}\left(\begin{array}{c}
a-\mu, b \\
c
\end{array} x^{-1}\right) \\
(x \in(-\infty, 0) \cup(1, \infty), \operatorname{Re} a>\operatorname{Re} \mu>0) .
\end{gathered}
$$

Combination with $[7,2.10(2)$ and (5)] (or with [18, (15.8.2)]) yields (4.7) for $x<0$. This, in its turn, implies by transformation of integration variable and by analytic continuation that

$$
\begin{aligned}
\int_{1}^{\infty} & { }_{2} F_{1}\left(\begin{array}{c}
a, b \\
c
\end{array} ; 1+t z\right) \frac{(t-1)^{\mu-1}}{\Gamma(\mu)} d t=\frac{\Gamma(a-\mu)}{\Gamma(a)} \frac{\Gamma(b-\mu)}{\Gamma(b)} \frac{\Gamma(c)}{\Gamma(c-\mu)} \\
& \times(-z)^{-\mu}{ }_{2} F_{1}\left(\begin{array}{c}
a-\mu, b-\mu \\
c-\mu
\end{array} 1+z\right) \quad(z \notin[0, \infty), \operatorname{Re} a>\operatorname{Re} \mu>0) .
\end{aligned}
$$

From that we see that (4.7) holds for $x<1$.

Case $\boldsymbol{a}-\boldsymbol{c}-\mathbf{c}$. By using (2.2) in (4.7) we arrive at

$$
\begin{gathered}
\int_{x}^{1}(1-y)^{a-\mu-1}{ }_{2} F_{1}\left(\begin{array}{c}
a, b \\
c
\end{array} ; y\right) \frac{(y-x)^{\mu-1}}{\Gamma(\mu)} d y=\frac{\Gamma(a-\mu)}{\Gamma(a)} \frac{\Gamma(c-b-\mu)}{\Gamma(c-b)} \frac{\Gamma(c)}{\Gamma(c-\mu)} \\
\times(1-x)^{a-1}{ }_{2} F_{1}\left(\begin{array}{c}
a-\mu, b \\
c-\mu
\end{array} ; x\right) \quad(x<1, \operatorname{Re} a, \operatorname{Re}(c-b)>\operatorname{Re} \mu>0) .
\end{gathered}
$$

Case $\boldsymbol{c}-$. By using (2.3) in (4.7) we arrive at

$$
\begin{gathered}
\int_{-\infty}^{x}(1-y)^{a+b-c}{ }_{2} F_{1}\left(\begin{array}{c}
a, b \\
c
\end{array} ; y\right) \frac{(x-y)^{\mu-1}}{\Gamma(\mu)} d y=\frac{\Gamma(c-a-\mu)}{\Gamma(c-a)} \frac{\Gamma(c-b-\mu)}{\Gamma(c-b)} \frac{\Gamma(c)}{\Gamma(c-\mu)} \\
\times(1-x)^{a+b-c+\mu}{ }_{2} F_{1}\left(\begin{array}{c}
a, b \\
c-\mu
\end{array} ; x\right) \quad(x<1, \operatorname{Re}(c-a), \operatorname{Re}(c-b)>\operatorname{Re} \mu>0)
\end{gathered}
$$

\subsection{Transmutation formulas}

Corresponding to case $c+$ above, we gave already (3.12), which gives rise to the transmutation formula (1.6), and by which a proof of (4.1) can be given. Analogues of (3.12) and (1.6) can be given for all cases above. These have the general form

$$
L_{a^{\prime}, b^{\prime}, c^{\prime} ; x}\left(\frac{w(y)}{v(x)}|x-y|^{\mu-1}\right)=L_{1-a, 1-b, 2-c ; y}\left(\frac{w(y) w_{2}(y)}{v(x) v_{2}(x)}|x-y|^{\mu-1}\right)
$$

and

$$
L_{a^{\prime}, b^{\prime}, c^{\prime} ; x}\left(\int_{I} f(y) \frac{w(y)}{v(x)}|x-y|^{\mu-1} d y\right)=\int_{I}\left(L_{a, b, c} f\right)(y) \frac{w(y) w_{2}(y)}{v(x) v_{2}(x)}|x-y|^{\mu-1} d y .
$$

The data to be used in these two formulas for the eight cases are specified in the table below. In general, $w(y)$ is a product of powers of $|y|$ and $1-y$, and $w_{2}(y)$ is equal to $|y|, 1-y$ or 1 . Similarly for $v(x)$ and $v_{2}(x)$, respectively. The variable $y$ in (4.12) ranges over an open interval $I$ which is also the integration interval in (4.13). The interval $I$ has endpoint $x$ at one side and endpoint $x_{0}=0,1$ or $-\infty$ at the other side. The variable $x$ in (4.12) and (4.13) ranges over some open subset $J$ of $\mathbb{R}$. The function $f$ in (4.13) should be in $C^{2}(J)$ and should moreover satisfy certain growth conditions at $x_{0}$, to be specified in a moment. The parameter $\mu$ can be arbitrarily complex in (4.12) but should satisfy $\operatorname{Re} \mu>2$ in (4.13). 


\begin{tabular}{|c|c|c|c|c|c|c|c|}
\hline case & $a^{\prime}$ & $b^{\prime}$ & $c^{\prime}$ & $x_{0}$ & $w(y) / v(x)$ & $w_{2}(y) / v_{2}(x)$ & $J$ \\
\hline$c+$ & $a$ & $b$ & $c+\mu$ & 0 & $|y|^{c-1} /|x|^{c+\mu-1}$ & & $(-\infty, 0) \cup(0,1)$ \\
\hline$a+, c+$ & $a+\mu$ & $b$ & $c+\mu$ & 0 & $\frac{|y|^{c-1}(1-y)^{b-c-\mu}}{|x|^{c+\mu-1}(1-x)^{b-c}}$ & $\frac{1-y}{1-x}$ & $(-\infty, 0) \cup(0,1)$ \\
\hline$a+, b+, c+$ & $a+\mu$ & $b+\mu$ & $c+\mu$ & 0 & $\frac{|y|^{c-1}(1-y)^{a+b-c}}{|x|^{c+\mu-1}(1-x)^{b-c}}$ & & $(-\infty, 0) \cup(0,1)$ \\
\hline$a-$ & $a-\mu$ & $b$ & $c$ & 0 & $|y|^{a-\mu-1} /|x|^{a-1}$ & $|y| /|x|$ & $(-\infty, 0) \cup(0,1)$ \\
\hline$a+$ & $a+\mu$ & $b$ & $c$ & 0 & $\frac{|y|^{c-a-\mu-1}(1-y)^{a+b-c}}{|x|^{c-a-1}(1-x)^{a+b-c+\mu}}$ & $|y| /|x|$ & $(-\infty, 0) \cup(0,1)$ \\
\hline$a-, b-, c-$ & $a-\mu$ & $b-\mu$ & $c-\mu$ & $-\infty$ & 1 & 1 & $(-\infty, 1)$ \\
\hline$a-, c-$ & $a-\mu$ & $b$ & $c-\mu$ & 1 & $\frac{(1-y)^{a-\mu-1}}{(1-x)^{a-1}}$ & $\frac{1-y}{1-x}$ & $(-\infty, 1)$ \\
\hline$c-$ & $a$ & $b$ & $c-\mu$ & $-\infty$ & $\frac{(1-y)^{a+b-c}}{(1-x)^{a+b-c+\mu}}$ & 1 & $(-\infty, 1)$ \\
\hline
\end{tabular}

Verification of (4.12) in the eight cases is by straightforward computation, possibly using computer algebra.

In order to find the growth conditions in (4.13) for $f$ at $x_{0}$ we recall that (4.13) is obtained from the string of equalities

$$
\begin{aligned}
& L_{a^{\prime}, b^{\prime}, c^{\prime} ; x}\left(\int_{I} f(y) \frac{w(y)}{v(x)}|x-y|^{\mu-1} d y\right)=\int_{I} f(y) L_{a^{\prime}, b^{\prime}, c^{\prime} ; x}\left(\frac{w(y)}{v(x)}|x-y|^{\mu-1}\right) d y \\
& \quad=\int_{I} f(y) L_{1-a, 1-b, 2-c ; y}\left(\frac{w(y) w_{2}(y)}{v(x) v_{2}(x)}|x-y|^{\mu-1}\right) d y \\
& =\int_{I}\left(L_{a, b, c} f\right)(y) \frac{w(y) w_{2}(y)}{v(x) v_{2}(x)}|x-y|^{\mu-1} d y .
\end{aligned}
$$

In the various steps we have to consider the singularities for $y$ at $x$ and at $x_{0}$. The first singularity is already taken into account by the condition $\operatorname{Re} \mu>2$. As for the singularity at $x_{0}$ the first and the third equality need extra assumptions (which will also imply that the four parts of the string are well defined). For the first equality we need

$$
f(y) w(y)(1+|y|)^{\operatorname{Re} \mu-1} \text { as a function of } y \text { is } L^{1} \text { at } y=x_{0} .
$$

For the third equality the needed assumptions follow from Lemma 3.2:

$$
\begin{aligned}
& \left.\begin{array}{l}
f^{\prime \prime}(y) y(1-y) w(y) w_{2}(y)(1+|y|)^{\operatorname{Re} \mu-1} \\
f^{\prime}(y) w(y) w_{2}(y)(1+|y|)^{\operatorname{Re} \mu} \\
f(y) y(1-y) \frac{d^{2}}{d y^{2}}\left(w(y) w_{2}(y)(1+|y|)^{\operatorname{Re} \mu-1}\right)
\end{array}\right\} \text { as functions of } y \text { are } L^{1} \text { at } y=x_{0}, \\
& f(y) \frac{d}{d y}\left(w(y) w_{2}(y)(1+|y|)^{\operatorname{Re} \mu}\right) \\
& f(y) w(y) w_{2}(y)(1+|y|)^{\operatorname{Re} \mu-1}
\end{aligned}
$$

and

$$
\left.\begin{array}{l}
f^{\prime}(y) y(1-y) w(y) w_{2}(y)(1+|y|)^{\operatorname{Re} \mu-1} \\
f(y) y(1-y) \frac{d}{d y}\left(w(y) w_{2}(y)(1+|y|)^{\operatorname{Re} \mu-1}\right) \\
f(y) w(y) w_{2}(y)(1+|y|)^{\operatorname{Re} \mu}
\end{array}\right\} \rightarrow 0 \text { as } y \rightarrow x_{0} .
$$


Thus, for given $f$, the identity (4.13) is settled under certain constraints for $a, b, c$ and $\mu$. But then the identity will be valid under relaxed constraints on $a, b, c$ and $\mu$ such that both sides of (4.13) are analytic in these four parameters, i.e., if $\operatorname{Re} \mu>2$ and

$$
\left.\begin{array}{l}
f^{\prime \prime}(y) y(1-y) w(y) w_{2}(y)(1+|y|)^{\operatorname{Re} \mu-1} \\
f^{\prime}(y) w(y) w_{2}(y)(1+|y|)^{\operatorname{Re} \mu} \\
f(y) w(y)(1+|y|)^{\operatorname{Re} \mu-1}
\end{array}\right\} \text { as functions of } y \text { are } L^{1} \text { at } y=x_{0}
$$

\section{$5 \quad$ Fractional integral transformations for the six solutions of the hypergeometric differential equation}

Formula (4.1) is a fractional integral transformation of type $c+$ for the solution $w_{1}$ of the hypergeometric differential equation. It turns out that for all six solutions $w_{i}$ there is such a transformation of the form $(.)^{c-1} w_{i}(. ; a, b, c) \rightarrow(.)^{c+\mu-1} w_{i}(. ; a, b, c+\mu)$. These can all be obtained by rewriting fractional integral transformations for ${ }_{2} F_{1}(a, b ; c ;$.$) of various types given$ in Section 4, namely types $c+; a-, b-, c-; a-; a-; c-; a+, b+, c+$, respectively. Here we list these formulas. Each formula is preceded by the formula number of the formula in Section 4 to which it reduces:

$$
\begin{aligned}
& \text { (4.1): } \quad \int_{0<y / x<1}|y|^{c-1} w_{1}(y ; a, b, c) \frac{(x-y)^{\mu-1}}{\Gamma(\mu)} d y=\frac{\Gamma(c)}{\Gamma(c+\mu)}|x|^{c+\mu-1} w_{1}(x ; a, b, c+\mu) \\
& (x \in(-\infty, 0) \cup(0,1), \operatorname{Re} c>0, \operatorname{Re} \mu>0) ; \\
& \text { (4.7): } \quad \int_{-\infty}^{x}|y|^{c-1} w_{2}(y ; a, b, c) \frac{(x-y)^{\mu-1}}{\Gamma(\mu)} d y=\frac{\Gamma(a-c-\mu+1)}{\Gamma(a-c+1)} \\
& \times \frac{\Gamma(b-c-\mu+1)}{\Gamma(b-c+1)} \frac{\Gamma(2-c)}{\Gamma(2-c-\mu)}|x|^{c+\mu-1} w_{2}(x ; a, b, c+\mu) \\
& (x \in(-\infty, 1), \operatorname{Re}(a-c+1), \operatorname{Re}(b-c+1)>\operatorname{Re} \mu>0) ; \\
& \text { (4.8): } \quad \int_{y / x>1}|y|^{c-1} w_{3}(y ; a, b, c) \frac{|x-y|^{\mu-1}}{\Gamma(\mu)} d y=\frac{\Gamma(a-c-\mu+1)}{\Gamma(a-c+1)}|x|^{c+\mu-1} w_{3}(x ; a, b, c+\mu) \\
& (x \in(-\infty, 0) \cup(1, \infty), \operatorname{Re}(a-c+1)>\operatorname{Re} \mu>0) ; \\
& \text { (4.8): } \quad \int_{y / x>1}|y|^{c-1} w_{4}(y ; a, b, c) \frac{|x-y|^{\mu-1}}{\Gamma(\mu)} d y=\frac{\Gamma(b-c-\mu+1)}{\Gamma(b-c+1)}|x|^{c+\mu-1} w_{4}(x ; a, b, c+\mu) \\
& (x \in(-\infty, 0) \cup(1, \infty), \operatorname{Re}(b-c+1)>\operatorname{Re} \mu>0) ; \\
& \text { (4.11): } \quad \int_{x}^{\infty} y^{c-1} w_{5}(y ; a, b, c) \frac{(y-x)^{\mu-1}}{\Gamma(\mu)} d y=\frac{\Gamma(b-c-\mu+1)}{\Gamma(b-c+1)} \\
& \times \frac{\Gamma(a-c-\mu+1)}{\Gamma(a-c+1)} \frac{\Gamma(a+b-c+1)}{\Gamma(a+b-c-\mu+1)} x^{c+\mu-1} w_{5}(x ; a, b, c+\mu) \\
& (x \in(0, \infty), \operatorname{Re}(b-c+1), \operatorname{Re}(a-c+1)>\operatorname{Re}(\mu)>0) ; \\
& \text { (4.3): } \quad \int_{0<\frac{1-y}{1-x}<1} y^{c-1} w_{6}(y ; a, b, c) \frac{|y-x|^{\mu-1}}{\Gamma(\mu)} d y \\
& =\frac{\Gamma(c-a-b+1)}{\Gamma(c-a-b+\mu+1)} x^{c+\mu-1} w_{6}(x ; a, b, c+\mu) \\
& (x \in(0,1) \cup(1, \infty), \operatorname{Re}(c-a-b+1)>0, \operatorname{Re} \mu>0) .
\end{aligned}
$$

It is a straightforward exercise to list also the fractional integral transformation formulas of the $w_{i}$ corresponding to the other seven types. We do not list all these formulas here, but 
only give their essential behaviour in the following table. Here an entry in $i$ th row, $j$ th column (place $(i, j)$ ) tells us that the transformation formula for $w_{j}$ of type given at $(i, 1)$ can be reduced to the transformation formula for $w_{1}$ of type given at $(i, j)$.

\begin{tabular}{|l||l|l|l|l|l|}
\hline$w_{1}$ & $w_{2}$ & $w_{3}$ & $w_{4}$ & $w_{5}$ & $w_{6}$ \\
\hline$c+$ & $a-, b-, c-$ & $a-$ & $a-$ & $c-$ & $a+, b+, c+$ \\
$a+, c+$ & $a-, c-$ & $a+, c+$ & $a-, c-$ & $a+$ & $a+$ \\
$a+, b+, c+$ & $c-$ & $a+$ & $a+$ & $a+, b+, c+$ & $c-$ \\
$a-$ & $a-$ & $a-, b-, c-$ & $c+$ & $a-, c-$ & $a+, c+$ \\
$a+$ & $a+$ & $a+, b+, c+$ & $c-$ & $a+, c+$ & $a-, c-$ \\
$a-, b-, c-$ & $c+$ & $a-$ & $a-$ & $a-, b-, c-$ & $c+$ \\
$a-, c-$ & $a+, c+$ & $a-, c-$ & $a+, c+$ & $a-$ & $a-$ \\
$c-$ & $a+, b+, c+$ & $a+$ & $a+$ & $c+$ & $a-, b-, c-$ \\
\hline
\end{tabular}

The cases $c+; c-; a-, b-, c-; a+, b+, c+$ occur 5 times, the cases $a+, c+; a-, c-6$ times, and the cases $a-; a+8$ times. That these numbers are not all equal will be caused by the symmetry in $a$ and $b$ of the hypergeometric function.

Some more examples from the above table which we need later (because of specialization to Euler type integral representations) are:

$$
\begin{aligned}
& \int_{0<\frac{y}{x}<1}|y|^{a-\mu-1} w_{2}(y ; a, b, c) \frac{|x-y|^{\mu-1}}{\Gamma(\mu)} d y=\frac{\Gamma(a-c-\mu+1)}{\Gamma(a-c+1)}|x|^{a-1} w_{2}(x ; a-\mu, b, c) \\
& (x \in(-\infty, 0) \cup(0,1), \operatorname{Re}(a-c+1)>\operatorname{Re} \mu>0), \\
& \int_{\frac{y}{x}>1} w_{4}(y ; a, b, c) \frac{|x-y|)^{\mu-1}}{\Gamma(\mu)} d y=\frac{\Gamma(b-\mu)}{\Gamma(b)} w_{4}(x ; a-\mu, b-\mu, c-\mu) \\
& (x \in(-\infty, 0) \cup(1, \infty), \operatorname{Re} b>\operatorname{Re} \mu>0), \\
& \int_{0<\frac{1-y}{1-x}<1} w_{6}(y ; a, b, c) \frac{|x-y|^{\mu-1}}{\Gamma(\mu)} d y=\frac{\Gamma(c-a-b+1)}{\Gamma(c-a-b+\mu+1)} w_{6}(x ; a-\mu, b-\mu, c-\mu) \\
& (x \in(0,1) \cup(1, \infty), \operatorname{Re}(c-a-b+1)>0, \operatorname{Re} \mu>0) .
\end{aligned}
$$

\section{Generalized Stieltjes transforms between solutions of the hypergeometric differential equation}

First we recall the proof of (3.22) as given by Karp \& Sitnik [12, Lemma 2]. Expand for $|z|<1$ the left-hand side of (3.22) as

$$
\sum_{k=0}^{\infty} \frac{(a)_{k}}{k !} z^{k} \int_{0}^{1} t^{d+e-b-c-1}(1-t)^{b+k-1}{ }_{2} F_{1}\left(\begin{array}{c}
d-c, e-c \\
d+e-b-c
\end{array} ; t\right) d t .
$$

By the limit case $x \uparrow 1$ of (1.5) this equals

$$
\sum_{k=0}^{\infty} \frac{(a)_{k}}{k !} z^{k} \frac{\Gamma(d+e-b-c) \Gamma(b+k)}{\Gamma(d+e-c+k)}{ }_{2} F_{1}\left(\begin{array}{c}
d-c, e-c \\
d+e-c+k
\end{array} ; 1\right) .
$$

By the Gauss summation formula [1, Theorem 2.2.2] we get

$$
\sum_{k=0}^{\infty} \frac{(a)_{k}}{k !} z^{k} \frac{\Gamma(d+e-b-c) \Gamma(b+k)}{\Gamma(d+e-c+k)} \frac{\Gamma(c+k) \Gamma(d+e-c+k)}{\Gamma(d+k) \Gamma(e+k)},
$$

which is equal to the right-hand side of (3.22). All steps can be rigorously justified because of the constraints in (3.22). 
We now list the generalized Stieltjes transforms mapping a solution $w_{i}$ to a solution $w_{j}$. They can all be obtained from the special case (3.23) of (3.22) by change of parameters, change of integration variable, and application of (2.2) and (2.3). In particular, (6.10) and (6.12) below are simple rewritings of (3.23) by a change of parameters. Also, (6.1) below is a rewriting of [8, $20.2(10)]$.

The formulas in the list are grouped in cases similar to the cases in Section 4. In all formulas the constraints are that $x$ is in an interval $(-\infty, 0),(0,1)$ or $(1, \infty)$ which does not coincide with the integration interval, and that the arguments of the three gamma functions in the numerator on the right-hand side have positive real parts.

\section{Case $c+$.}

$$
\begin{aligned}
\int_{-\infty}^{0} & (-y)^{c-1} w_{1}(y ; a, b, c)(x-y)^{\mu-1} d y \\
& =\frac{\Gamma(a-c-\mu+1) \Gamma(b-c-\mu+1) \Gamma(c)}{\Gamma(a+b-c-\mu+1) \Gamma(1-\mu)} x^{c-1+\mu} w_{5}(x ; a, b, c+\mu), \\
\int_{1}^{\infty} y^{c-1} w_{6}(y ; a, b, c)(y-x)^{\mu-1} d y & \\
\quad= & \frac{\Gamma(a-c-\mu+1) \Gamma(b-c-\mu+1) \Gamma(c-a-b+1)}{\Gamma(2-c-\mu) \Gamma(1-\mu)}|x|^{c+\mu-1} w_{2}(x ; a, b, c+\mu) .
\end{aligned}
$$

\section{Case $a+, c+$.}

$$
\begin{aligned}
\int_{0}^{1} y^{c-1}(1-y)^{b-c-\mu} w_{1}(y ; a, b, c)|x-y|^{\mu-1} d y \\
=\frac{\Gamma(-a-\mu+1) \Gamma(b-c-\mu+1) \Gamma(c)}{\Gamma(b-a-\mu+1) \Gamma(1-\mu)} \\
\quad \times|x|^{c+\mu-1}|x-1|^{b-c} w_{4}(x ; a+\mu, b, c+\mu), \\
\int_{1}^{\infty} y^{c-1}(y-1)^{b-c-\mu} w_{3}(y ; a, b, c)(y-x)^{\mu-1} d y \\
=\frac{\Gamma(-a-\mu+1) \Gamma(b-c-\mu+1) \Gamma(a-b+1)}{\Gamma(-c-\mu+2) \Gamma(1-\mu)} \\
\quad \times|x|^{c+\mu-1}|x-1|^{b-c} w_{2}(x ; a+\mu, b, c+\mu) .
\end{aligned}
$$

\section{Case $b+, c+$.}

$$
\begin{aligned}
\int_{0}^{1} y^{c-1}(1-y)^{a-c-\mu} w_{1}(y ; a, b, c)|x-y|^{\mu-1} d y \\
=\frac{\Gamma(-b-\mu+1) \Gamma(a-c-\mu+1) \Gamma(c)}{\Gamma(a-b-\mu+1) \Gamma(1-\mu)} \\
\quad \times|x|^{c+\mu-1}|x-1|^{a-c} w_{3}(x ; a, b+\mu, c+\mu), \\
\int_{1}^{\infty} y^{c-1}(y-1)^{a-c-\mu} w_{4}(y ; a, b, c)|x-y|^{\mu-1} d y \\
=\frac{\Gamma(-b-\mu+1) \Gamma(a-c-\mu+1) \Gamma(b-a+1)}{\Gamma(-c-\mu+2) \Gamma(1-\mu)} \\
\quad \times|x|^{c+\mu-1}|x-1|^{a-c} w_{2}(x ; a, b+\mu, c+\mu) .
\end{aligned}
$$


Case $a+, b+, c+$.

$$
\begin{aligned}
& \int_{-\infty}^{0}(-y)^{c-1}(1-y)^{a+b-c} w_{1}(x ; a, b, c)(x-y)^{\mu-1} d y \\
&= \frac{\Gamma(-a-\mu+1) \Gamma(-b-\mu+1) \Gamma(c)}{\Gamma(c-a-b-\mu+1) \Gamma(1-\mu)} \\
& \quad \times x^{c+\mu-1}(1-x)^{a+b-c+\mu} w_{6}(x ; a+\mu, b+\mu, c+\mu), \\
& \int_{1}^{\infty} y^{c-1}(y-1)^{a+b-c} w_{5}(y ; a, b, c)(y-x)^{\mu-1} d y \\
&=\frac{\Gamma(-a-\mu+1) \Gamma(-b-\mu+1) \Gamma(a+b-c+1)}{\Gamma(2-c-\mu) \Gamma(1-\mu)} \\
& \quad \times|x|^{c+\mu-1}(1-x)^{a+b-c+\mu} w_{2}(x ; a+\mu, b+\mu, c+\mu) .
\end{aligned}
$$

\section{Case $a-$.}

$$
\begin{gathered}
\int_{-\infty}^{0}(-y)^{a-\mu-1} w_{4}(y ; a, b, c)(x-y)^{\mu-1} d y \\
\quad=\frac{\Gamma(a-\mu) \Gamma(a-c-\mu+1) \Gamma(b-a+1)}{\Gamma(a+b-c-\mu+1) \Gamma(1-\mu)} x^{a-1} w_{5}(x ; a-\mu, b, c), \\
\int_{0}^{1} y^{a-\mu-1} w_{6}(y ; a, b, c)|x-y|^{\mu-1} d y \\
\quad=\frac{\Gamma(a-c-\mu+1) \Gamma(a-\mu) \Gamma(c-a-b+1)}{\Gamma(a-b-\mu+1) \Gamma(1-\mu)}|x|^{a-1} w_{3}(x ; a-\mu, b, c) .
\end{gathered}
$$

\section{Case $b-$.}

$$
\begin{gathered}
\int_{-\infty}^{0}(-y)^{b-\mu-1} w_{3}(y ; a, b, c)(x-y)^{\mu-1} d y \\
=\frac{\Gamma(b-\mu) \Gamma(b-c-\mu+1) \Gamma(a-b+1)}{\Gamma(a+b-c-\mu+1) \Gamma(1-\mu)} x^{b-1} w_{5}(x ; a, b-\mu, c), \\
\int_{0}^{1} y^{b-\mu-1} w_{6}(y ; a, b, c)|x-y|^{\mu-1} d y \\
\quad=\frac{\Gamma(b-c-\mu+1) \Gamma(b-\mu) \Gamma(c-a-b+1)}{\Gamma(b-a-\mu+1) \Gamma(1-\mu)}|x|^{b-1} w_{4}(x ; a, b-\mu, c) .
\end{gathered}
$$

\section{Case $a+$.}

$$
\begin{gathered}
\int_{-\infty}^{0}(-y)^{c-a-\mu-1}(1-y)^{a+b-c} w_{3}(y ; a, b, c)(x-y)^{\mu-1} d y \\
=\frac{\Gamma(-a-\mu+1) \Gamma(c-a-\mu) \Gamma(a-b+1)}{\Gamma(c-a-b-\mu+1) \Gamma(1-\mu)} x^{c-a-1}(1-x)^{a+b-c+\mu} w_{6}(x ; a+\mu, b, c), \\
\int_{0}^{1} y^{c-a-\mu-1}(1-y)^{a+b-c} w_{5}(y ; a, b, c)|x-y|^{\mu-1} d y \\
=\frac{\Gamma(-a-\mu+1) \Gamma(c-a-\mu) \Gamma(a+b-c+1)}{\Gamma(b-a-\mu+1) \Gamma(1-\mu)} \\
\quad \times|x|^{c-a-1}|1-x|^{a+b-c-\mu} w_{4}(x ; a+\mu, b, c) .
\end{gathered}
$$




\section{Case $b+$.}

$$
\begin{gathered}
\int_{-\infty}^{0}(-y)^{c-b-\mu-1}(1-y)^{a+b-c} w_{4}(y ; a, b, c)(x-y)^{\mu-1} d y \\
=\frac{\Gamma(-b-\mu+1) \Gamma(c-b-\mu) \Gamma(b-a+1)}{\Gamma(c-a-b-\mu+1) \Gamma(1-\mu)} \\
\quad \times x^{c-b-1}(1-x)^{a+b-c+\mu} w_{6}(x ; a, b+\mu, c), \\
\int_{0}^{1} y^{c-b-\mu-1}(1-y)^{a+b-c} w_{5}(y ; a, b, c)|x-y|^{\mu-1} d y \\
=\frac{\Gamma(-b-\mu+1) \Gamma(c-b-\mu) \Gamma(a+b-c+1)}{\Gamma(a-b-\mu+1) \Gamma(1-\mu)} \\
\quad \times|x|^{c-b-1}|1-x|^{a+b-c-\mu} w_{3}(x ; a, b+\mu, c) .
\end{gathered}
$$

\section{Case $a-, b-, c-$.}

$$
\begin{aligned}
& \int_{-\infty}^{0} w_{2}(y ; a, b, c)(x-y)^{\mu-1} d y \\
&=\frac{\Gamma(a-\mu) \Gamma(b-\mu) \Gamma(2-c)}{\Gamma(a+b-c-\mu+1) \Gamma(1-\mu)} w_{5}(x ; a-\mu, b-\mu, c-\mu), \\
& \int_{1}^{\infty} w_{6}(y ; a, b, c)(y-x)^{\mu-1} d y \\
& \quad=\frac{\Gamma(a-\mu) \Gamma(b-\mu) \Gamma(c-a-b+1)}{\Gamma(c-\mu) \Gamma(1-\mu)} w_{1}(x ; a-\mu, b-\mu, c-\mu) .
\end{aligned}
$$

\section{Case $a-, c-$.}

$$
\begin{aligned}
& \int_{0}^{1}(1-y)^{a-\mu-1} w_{2}(y ; a, b, c)|x-y|^{\mu-1} d y \\
& \quad=\frac{\Gamma(c-b-\mu) \Gamma(a-\mu) \Gamma(2-c)}{\Gamma(a-b-\mu+1) \Gamma(1-\mu)}|x-1|^{a-1} w_{3}(x ; a-\mu, b, c-\mu), \\
& \int_{1}^{\infty}(y-1)^{a-\mu-1} w_{4}(y ; a, b, c)(y-x)^{\mu-1} d y \\
& \quad=\frac{\Gamma(c-b-\mu) \Gamma(a-\mu) \Gamma(b-a+1)}{\Gamma(c-\mu) \Gamma(1-\mu)}|1-x|^{a-1} w_{1}(x ; a-\mu, b, c-\mu) .
\end{aligned}
$$

\section{Case $b-, c-$.}

$$
\begin{aligned}
& \int_{0}^{1}(1-y)^{b-\mu-1} w_{2}(y ; a, b, c)|x-y|^{\mu-1} d y \\
& \quad=\frac{\Gamma(c-a-\mu) \Gamma(b-\mu) \Gamma(2-c)}{\Gamma(b-a-\mu+1) \Gamma(1-\mu)}|x-1|^{b-1} w_{4}(x ; a, b-\mu, c-\mu), \\
& \int_{1}^{\infty}(y-1)^{b-\mu-1} w_{3}(y ; a, b, c)(y-x)^{\mu-1} d y \\
& \quad=\frac{\Gamma(c-a-\mu) \Gamma(b-\mu) \Gamma(a-b+1)}{\Gamma(c-\mu) \Gamma(1-\mu)}|1-x|^{b-1} w_{1}(x ; a, b-\mu, c-\mu) .
\end{aligned}
$$




\section{Case $c-$.}

$$
\begin{aligned}
\int_{-\infty}^{0} & (1-y)^{a+b-c} w_{2}(y ; a, b, c)(x-y)^{\mu-1} d y \\
\quad & =\frac{\Gamma(c-a-\mu) \Gamma(c-b-\mu) \Gamma(2-c)}{\Gamma(c-a-b-\mu+1) \Gamma(1-\mu)}|1-x|^{a+b-c+\mu} w_{6}(x ; a, b, c-\mu), \\
\int_{1}^{\infty} & (y-1)^{a+b-c} w_{5}(y ; a, b, c)(y-x)^{\mu-1} d y \\
\quad= & \frac{\Gamma(c-a-\mu) \Gamma(c-b-\mu) \Gamma(a+b-c+1)}{\Gamma(c-\mu) \Gamma(1-\mu)}(1-x)^{a+b-c+\mu} w_{1}(x ; a, b, c-\mu) .
\end{aligned}
$$

We summarize the results of the above list in the following table. In the box in row $w_{i}$ and column $w_{j}$ the type is given of the generalized Stieltjes transform sending $w_{i}$ to $w_{j}$.

\begin{tabular}{|l||l|l|l|l|l|l|}
\hline & $w_{1}$ & $w_{2}$ & $w_{3}$ & $w_{4}$ & $w_{5}$ & $w_{6}$ \\
\hline \hline$w_{1}$ & & & $b+, c+$ & $a+, c+$ & $c+$ & $a+, b+, c+$ \\
\hline$w_{2}$ & & & $a-, c-$ & $b-, c-$ & $a-, b-, c-$ & $c-$ \\
\hline$w_{3}$ & $b-, c-$ & $a+, c+$ & & & $b-$ & $a+$ \\
\hline$w_{4}$ & $a-, c-$ & $b+, c+$ & & & $a-$ & $b+$ \\
\hline$w_{5}$ & $c-$ & $a+, b+, c+$ & $b+$ & $a+$ & & \\
\hline$w_{6}$ & $a-, b-, c-$ & $c+$ & $a-$ & $b-$ & & \\
\hline
\end{tabular}

Due to the denominator factor $\Gamma(1-\mu)$ all generalized Stieltjes transforms above become zero if $\mu$ is a positive integer satisfying the constraints.

\section{$7 \quad$ Euler type integral representations}

Below we give the explicit Euler type integral representations which have form (1.4) or (3.21).

Euler type integrals with integrand $|x|^{1-c}|y|^{b-1}|1-y|^{-a}|x-y|^{c-b-1}$.

$$
\begin{aligned}
w_{1}(x ; a, b, c)= & \frac{\Gamma(c)}{\Gamma(b) \Gamma(c-b)}|x|^{1-c} \int_{0<y / x<1}|y|^{b-1}(1-y)^{-a}|x-y|^{c-b-1} d y \\
& (x \in(-\infty, 0) \cup(0,1), \operatorname{Re} c>\operatorname{Re} b>0), \\
w_{2}(x ; a, b, c)= & \frac{\Gamma(2-c)}{\Gamma(a-c+1) \Gamma(1-a)}|x|^{1-c} \int_{1}^{\infty} y^{b-1}(y-1)^{-a}(y-x)^{c-b-1} d y \\
& (x \in(-\infty, 0) \cup(0,1), \operatorname{Re}(c-1)<\operatorname{Re} a<1), \\
w_{3}(x ; a, b, c)= & \frac{\Gamma(a-b+1)}{\Gamma(a-c+1) \Gamma(c-b)}|x|^{1-c} \int_{y / x>1}|y|^{b-1}|y-1|^{-a}|y-x|^{c-b-1} d y \\
& (x \in(-\infty, 0) \cup(1, \infty), \operatorname{Re}(a-c+1)>0, \operatorname{Re}(c-b)>0), \\
w_{4}(x ; a, b, c)= & \frac{\Gamma(b-a+1)}{\Gamma(-a) \Gamma(b+1)}|x|^{1-c} \int_{0}^{1} y^{b-1}(1-y)^{-a}|x-y|^{c-b-1} d y \\
& (x \in(-\infty, 0) \cup(1, \infty), \operatorname{Re} a<0, \operatorname{Re} b>-1), \\
w_{5}(x ; a, b, c)= & \frac{\Gamma(a+b-c+1)}{\Gamma(a-c+1) \Gamma(b)} x^{1-c} \int_{-\infty}^{0}(-y)^{b-1}(1-y)^{-a}(x-y)^{c-b-1} d y \\
& (x \in(0, \infty), \operatorname{Re} b>0, \operatorname{Re}(a-c+1)>0), \\
w_{6}(x ; a, b, c)= & \frac{\Gamma(c-a-b+1)}{\Gamma(1-a) \Gamma(c-b)} x^{1-c} \int_{0<\frac{1-y}{1-x}<1} y^{b-1}(1-y)^{-a}|x-y|^{c-b-1} d y \\
& (x \in(0,1) \cup(1, \infty), \operatorname{Re}(c-b)>0, \operatorname{Re} a<1) .
\end{aligned}
$$


Euler type integral transformations with integrand $|y|^{a-c}|1-y|^{c-b-1}|x-y|^{-a}$.

$$
\begin{aligned}
& w_{1}(x ; a, b, c)=\frac{\Gamma(c)}{\Gamma(b) \Gamma(c-b)} \int_{1}^{\infty} y^{a-c}(y-1)^{c-b-1}(y-x)^{-a} d y \\
& (x<1, \operatorname{Re} c>\operatorname{Re} b>0), \\
& w_{2}(x ; a, b, c)=\frac{\Gamma(2-c)}{\Gamma(a-c+1) \Gamma(1-a)} \int_{0<y / x<1}|y|^{a-c}(1-y)^{c-b-1}|x-y|^{-a} d y \\
& (x \in(-\infty, 0) \cup(0,1), 2>\operatorname{Re}(a+1)>\operatorname{Re} c), \\
& w_{3}(x ; a, b, c)=\frac{\Gamma(a-b+1)}{\Gamma(a-c+1) \Gamma(c-b)} \int_{0}^{1} y^{a-c}(1-y)^{c-b-1}|x-y|^{-a} d y \\
& (x \in(-\infty, 0) \cup(1, \infty), \operatorname{Re}(a+1)>\operatorname{Re} c>\operatorname{Re} b), \\
& w_{4}(x ; a, b, c)=\frac{\Gamma(b-a+1)}{\Gamma(-a) \Gamma(b+1)} \int_{y / x>1}|y|^{a-c}|y-1|^{c-b-1}|y-x|^{-a} d y \\
& (x \in(-\infty, 0) \cup(1, \infty), \operatorname{Re} a<1, \operatorname{Re} b>0), \\
& w_{5}(x ; a, b, c)=\frac{\Gamma(a+b-c+1)}{\Gamma(a-c+1) \Gamma(b)} \int_{-\infty}^{0}(-y)^{a-c}(1-y)^{c-b-1}(x-y)^{-a} d y \\
& (x \in(0, \infty), \operatorname{Re} b>0, \operatorname{Re}(a+1)>\operatorname{Re} c), \\
& w_{6}(x ; a, b, c)=\frac{\Gamma(c-a-b+1)}{\Gamma(1-a) \Gamma(c-b)} \int_{0<\frac{1-y}{1-x}<1}|y|^{a-c}|1-y|^{c-b-1}|x-y|^{-a} d y \\
& (x \in(0,1) \cup(1, \infty), \operatorname{Re}(c-b)>0, \operatorname{Re} a<1) .
\end{aligned}
$$

The integrals in (7.1), (7.3), (7.6) (for $\left.w_{1}, w_{3}, w_{6}\right)$ and in (7.8), (7.10), (7.12) (for $w_{2}, w_{4}, w_{6}$ ) are of fractional integral type. Formulas (7.3), (7.8), (7.10) and (7.12) can be reduced to (1.1) by a change of integration variable, while (7.1) is a rewritten version of (1.1). Each of the six formulas (7.1)-(7.6) is paired with one of the six formulas (7.7)-(7.12) by a transformation of integration variable which involves $x$. The pairing is as follows:

$$
\begin{aligned}
& (7.1) \leftrightarrow(7.9), \quad(7.2) \leftrightarrow(7.10), \quad(7.3) \leftrightarrow(7.7), \\
& (7.4) \leftrightarrow(7.8), \quad(7.5) \leftrightarrow(7.11), \quad(7.6) \leftrightarrow(7.12) .
\end{aligned}
$$

In 1874 Letnikov [14, (11), (13), (16), (17), (21), (22)] (see also Sostak [23, Section 4]) already gave the Euler type integral representations (7.8), (7.12), (7.10), (7.1), (7.6), (7.3), respectively, as solutions of the hypergeometric differential equation [14, p. 115, (A)]. In order to arrive at these results he essentially considered the fractional integral operator as a transmutation operator with respect to the hypergeometric differential operator.

\begin{tabular}{|lllll|}
\hline int. rep. & transform & from & to & case \\
\hline \hline$(7.1)$ & $(5.1)$ & $w_{1}$ & $w_{1}$ & $c+$ \\
$(7.2)$ & $(6.4)$ & $w_{3}$ & $w_{2}$ & $a+, c+$ \\
$(7.3)$ & $(5.3)$ & $w_{3}$ & $w_{3}$ & $c+$ \\
$(7.4)$ & $(6.3)$ & $w_{1}$ & $w_{4}$ & $a+, c+$ \\
$(7.5)$ & $(6.1)$ & $w_{1}$ & $w_{5}$ & $c+$ \\
$(7.6)$ & $(5.6)$ & $w_{6}$ & $w_{6}$ & $c+$ \\
\hline$(7.7)$ & $(6.20)$ & $w_{4}$ & $w_{1}$ & $a-, c-$ \\
$(7.8)$ & $(5.7)$ & $w_{2}$ & $w_{2}$ & $a-$ \\
$(7.9)$ & $(6.10)$ & $w_{6}$ & $w_{3}$ & $a-$ \\
$(7.10)$ & $(5.8)$ & $w_{4}$ & $w_{4}$ & $a-, b-, c-$ \\
$(7.11)$ & $(6.9)$ & $w_{4}$ & $w_{5}$ & $a-$ \\
$(7.12)$ & $(5.9)$ & $w_{6}$ & $w_{6}$ & $a-, b-, c-$ \\
\hline
\end{tabular}


The above table gives for each Euler type integral representation in the first column the transformation formula in the second column from which it can be obtained by specialization of parameters ( $b=c$ in the first six rows and $a=-1$ in the last six rows). The transformation formula sends $w_{i}$ in the third column to $w_{j}$ in the fourth column. Its case is given in the fifth column. Formula (7.5) is essentially the same as $[8,14.4(9)]$.

\section{Generalized Stieltjes transform and fractional integral transform combined}

As observed in [8, p. 213], generalized Stieltjes transforms of different order are connected with each other by fractional integration. Formula (8.1) below was essentially given there, and later given with proof in [10, Theorem 9]. Some related identities can also be proved:

Proposition 8.1. Let $\operatorname{Re}(1-\nu)>\operatorname{Re} \mu>0$. Assume that $f \in L_{\mathrm{loc}}^{1}((m, M))$ and that the integrals on the right-hand side of the four identities below converge absolutely. Then

$$
\begin{aligned}
& \int_{-\infty}^{x}\left(\int_{m}^{M} f(z) \frac{\Gamma(1-\nu)}{(z-y)^{1-\nu}} d z\right) \frac{(x-y)^{\mu-1}}{\Gamma(\mu)} d y=\int_{m}^{M} f(z) \frac{\Gamma(1-\mu-\nu)}{(z-x)^{1-\mu-\nu}} d z \quad(x<m), \\
& \int_{x}^{\infty}\left(\int_{m}^{M} f(z) \frac{\Gamma(1-\nu)}{(y-z)^{1-\nu}} d z\right) \frac{(y-x)^{\mu-1}}{\Gamma(\mu)} d y=\int_{m}^{M} f(z) \frac{\Gamma(1-\mu-\nu)}{(x-z)^{1-\mu-\nu}} d z \quad(M<x),(8.2) \\
& \int_{m}^{\infty}\left(\int_{m}^{y} f(z) \frac{(y-z)^{\mu-1}}{\Gamma(\mu)} d z\right) \frac{\Gamma(1-\nu)}{(x-y)^{1-\nu}} d y=\int_{m}^{\infty} f(z) \frac{\Gamma(1-\mu-\nu)}{(z-x)^{1-\mu-\nu}} d z \quad(x<m), \\
& \int_{-\infty}^{M}\left(\int_{y}^{M} f(z) \frac{(z-y)^{\mu-1}}{\Gamma(\mu)} d z\right) \frac{\Gamma(1-\nu)}{(y-x)^{1-\nu}} d y=\int_{-\infty}^{M} f(z) \frac{\Gamma(1-\mu-\nu)}{(x-z)^{1-\mu-\nu}} d z \quad(x>M) .
\end{aligned}
$$

The proofs are immediate, by the Fubini theorem and by a version [18, (5.12.3)] of the beta integral. Furthermore, (8.2) is an immediate consequence of (8.1), and similarly (8.4) of (8.3).

Examples of formulas (8.1)-(8.4) can be found by combining suitable fractional integral formulas in Section 5 with suitable generalized Stieltjes transform formulas in Section 6. For instance:

- In (8.1) let $m=1, M=\infty, f(z)=z^{c-1} w_{6}(z ; a, b, c)$ and use (5.2) and (6.2).

- In (8.2) let $m=-\infty, M=0, f(z)=(-z)^{c-1} w_{1}(z ; a, b, c)$ and use (5.5) and (6.1).

- In (8.3) let $m=1, f(z)=z^{c-1} w_{6}(z ; a, b, c)$ and use (5.6) and (6.2).

- In (8.4) let $M=0, f(z)=(-z)^{c-1} w_{1}(z ; a, b, c)$ and use (5.1) and (6.1).

\section{Acknowledgements}

I am very grateful to Sergei Sitnik for his comments, in particular about Letnikov's paper [14] from 1874. Thanks also to Dmitry Karp for helpful comments. Furthermore, the paper took profit from comments and lists of typos in referees' reports.

\section{References}

[1] Andrews G.E., Askey R., Roy R., Special functions, Encyclopedia of Mathematics and its Applications, Vol. 71, Cambridge University Press, Cambridge, 1999.

[2] Askey R., Orthogonal polynomials and special functions, Society for Industrial and Applied Mathematics, Philadelphia, Pa., 1975.

[3] Askey R., Fitch J., Integral representations for Jacobi polynomials and some applications, J. Math. Anal. Appl. 26 (1969), 411-437. 
[4] Bateman H., The solution of linear differential equations by means of definite integrals, Trans. Cambridge Philos. Soc. 21 (1909), 171-196.

[5] Camporesi R., The biradial Paley-Wiener theorem for the Helgason Fourier transform on Damek-Ricci spaces, J. Funct. Anal. 267 (2014), 428-451.

[6] Dereziński J., Hypergeometric type functions and their symmetries, Ann. Henri Poincaré 15 (2014), 15691653, arXiv:1305.3113.

[7] Erdélyi A., Magnus W., Oberhettinger F., Tricomi F.G., Higher transcendental functions, Vol. I, Mc-Graw Hill, New York, 1953.

[8] Erdélyi A., Magnus W., Oberhettinger F., Tricomi F.G., Higher transcendental functions, Vol. II, Mc-Graw Hill, New York, 1953.

[9] Flanders H., Differentiation under the integral sign, Amer. Math. Monthly 80 (1973), 615-627, Correction, Amer. Math. Monthly 81 (1974), 145.

[10] Karp D., Prilepkina E., Generalized Stieltjes functions and their exact order, J. Class. Anal. 1 (2012), 53-74.

[11] Karp D., Prilepkina E., Hypergeometric functions as generalized Stieltjes transforms, J. Math. Anal. Appl. 393 (2012), 348-359, arXiv:1112.5769.

[12] Karp D., Sitnik S.M., Inequalities and monotonicity of ratios for generalized hypergeometric function, $J . A p$ prox. Theory 161 (2009), 337-352, math.CA/0703084.

[13] Kodavanji S., Rathie A.K., Paris R.B., A derivation of two transformation formulas contiguous to that of Kummer's second theorem via a differential equation approach, arXiv:1501.06173.

[14] Letnikov A.V., Research related to the theory of integrals of the form $\int_{0}^{x}(x-u)^{p-1} f(u) d u$. Chapter III. Application to the integration of certain differential equations, Mat. Sb. 7 (1874), 111-205 (in Russian).

[15] Lions J.L., Opérateurs de Delsarte et problèmes mixtes, Bull. Soc. Math. France 84 (1956), 9-95.

[16] Miller K.S., Ross B., An introduction to the fractional calculus and fractional differential equations, A Wiley-Interscience Publication, John Wiley \& Sons, Inc., New York, 1993.

[17] Miller Jr. W., Lie theory and generalizations of the hypergeometric functions, SIAM J. Appl. Math. 25 (1973), 226-235.

[18] Olver F.W.J., Lozier D.W., Boisvert R.F., Clark C.W. (Editors), NIST handbook of mathematical functions, U.S. Department of Commerce National Institute of Standards and Technology, Washington, DC, Cambridge University Press, Cambridge, 2010, available at http://dlmf.nist.gov.

[19] Rainville E.D., Special functions, The Macmillan Co., New York, 1960.

[20] Saito M., Symmetry algebras of normal $\mathcal{A}$-hypergeometric systems, Hokkaido Math. J. 25 (1996), 591-619.

[21] Sitnik S.M., Transmutations and applications: a survey, arXiv:1012.3741 (in Russian).

[22] Sitnik S.M., Buschman-Erdelyi transmutations, classification and applications, arXiv:1304.2114.

[23] Sostak R.Ya., Aleksei Vasilevic Letnikov, Istor.-Mat. Issled. 5 (1952), 167-238 (in Russian).

[24] Swathi M., Rathie A.K., Paris R.B., A derivation of two quadratic transformations contiguous to that of Gauss via a differential equation approach, arXiv:1411.5262.

[25] Szegö G., Orthogonal polynomials, American Mathematical Society, Colloquium Publications, Vol. 23, 4th ed., Amer. Math. Soc., Providence, R.I., 1975.

[26] Widder D.V., The Stieltjes transform, Trans. Amer. Math. Soc. 43 (1938), 7-60. 